\title{
The costs of positon, velocity and force requirements in optimal control induce triphasic muscle activation during reaching movement
}

Yuki Ueyama ( $\square$ ueyama@nda.ac.jp)

National Defense Academy of Japan

\section{Research Article}

Keywords: Motor control, Musculoskeletal model, EMG, Muscle coordination, Optimal feedback control, Cost function

Posted Date: April 22nd, 2021

DOI: https://doi.org/10.21203/rs.3.rs-311040/v1

License: (9) This work is licensed under a Creative Commons Attribution 4.0 International License.

Read Full License

Version of Record: A version of this preprint was published at Scientific Reports on August 19th, 2021. See the published version at https://doi.org/10.1038/s41598-021-96084-2. 


\title{
The costs of positon, velocity and force requirements in optimal control induce triphasic muscle activation during reaching movement
}

\author{
Yuki Ueyama \\ Department of Mechanical Engineering, National Defense Academy of Japan, Yokosuka, \\ Kanagawa, Japan
}

Corresponding author: Yuki Ueyama

E-mail: ueyama@nda.ac.jp

Phone: +81-46-841-3810

ORCD ID: 0000-0002-1415-8875

\section{Abstract}

The nervous system activates a pair of agonist and antagonist muscles to determine the muscle activation pattern for a desired movement. Although there is a problem with redundancy, it is solved immediately, and movements are generated with characteristic muscle activation patterns in which antagonistic muscle pairs show alternate bursts with a triphasic shape. To investigate the requirements for deriving this pattern, this study simulated arm movement numerically by adopting a musculoskeletal arm model and an optimal control based on the minimization of neural input. The simulation reproduced the triphasic electromyogram (EMG) pattern observed in a reaching movement using a cost function that considered three terms: end-point position, velocity, and force required. The first, second and third bursts of muscle activity were generated by the cost terms of position, velocity and force, respectively. Thus we concluded that the costs of position, velocity and force requirements in optimal control can induce triphasic EMG patterns. Therefore we suggest that the nervous system may control the body by using 
an optimal control mechanism that adopts the costs of position, velocity and force required, which serve to initiate, decelerate and stabilize movement, respectively.

Keywords: Motor control, Musculoskeletal model, EMG, Muscle coordination, Optimal feedback control, Cost function 


\section{Introduction}

Mammalian biomechanical motor control comprises multiple joints and muscles that form redundant systems with multiple degrees of freedom. To move the nervous system must overcome the problem of redundancy and determine a movement trajectory and muscle activation pattern that involves pairs of agonist and antagonist muscles. The muscle activation pattern (i.e. the electromyogram, EMG) is equivalent to other movements loaded by an external force when the movements are identical kinematically and the dynamics are well learned, even if the amount of muscle activation is increased ${ }^{1}$. In both single-joint and multi-joint reaching movements, the agonist and antagonist muscles typically burst alternately in triphasic patterns ${ }^{2,3}$. Thus the muscles seem to be tuned selectively in accordance with the movement direction, similar to isometric force production ${ }^{4}$. Then the agonist and antagonist muscles are activated alternately in a triphasic pattern (Figure 1). An agonist muscles are strongly activated at movement onset (Figure 1, AG1). Next, the antagonist muscles show single peaks at the midpoint of the movement (Figure 1, ANT). The timing is synchronized with the peaks in the velocity profiles. Subsequently, the agonist muscles are activated again, albeit weakly

(Figure 1, AG2). The AG1 and ANT burst pair is thought to determine the increase and decrease in acceleration, respectively, and the AG2 burst increases deceleration ${ }^{5}$ or dampens the oscillations that might occur at the end of the movement ${ }^{6}$. Thus the triphasic EMG pattern does not relate directly to the movement amplitude, speed or duration, but to the acceleration and deceleration ${ }^{7}$. Such a simple, general relation between the EMG and movement implies that the nervous system can simply determine the muscle activation patterns needed to produce movements with desired 
characteristics ${ }^{8}$. Indeed single-neuron and population-level activity in the primary motor cortex (M1) also show triphasic changes in temporal pattern and instantaneous directionality similar to the $\mathrm{EMG}^{9,10}$, although it is thought that the neurons represent the speed and direction of hand motion ${ }^{11}$.

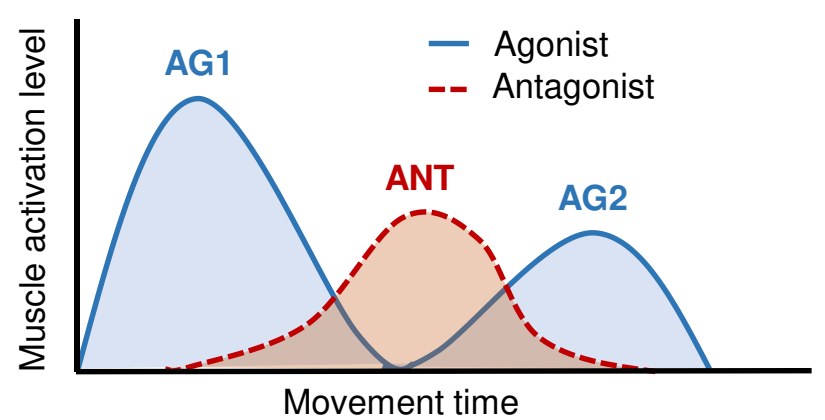

Figure 1. Triphasic muscle activation pattern during reaching movement. AG1, AG2 and ANT indicate the sequence of bursts for the pair of agonist (AG1 and AG2) and antagonist (ANT) muscles.

In the computational motor control domain, optimal feedback control (OFC) theory has been proposed ${ }^{12-14}$, which predicts not only various movement phenomena, such as obstacle avoidance ${ }^{15}$, adaptation to novel tasks ${ }^{16}$, stiffness modulation ${ }^{17}$ and the manipulation of complex objects ${ }^{18}$, but also neural representation in M1 ${ }^{19}$. An optimal control framework using an OFC-like cost function based on minimization of neural input can also reproduce muscle co-contraction depending on the task; this is impossible using previous models ${ }^{20,21}$, although most motor-planning models, such as the minimum-jerk ${ }^{22}$ and minimum-torque-change ${ }^{23}$ models, are thought to be unable to predict such specific muscle actions ${ }^{24}$. In neural studies of primates, M1 may provide a neural substrate for integrating shoulder and elbow motion information into joint torque 
for fast feedback control ${ }^{25,26}$. The neural activity in M1 is thought to be optimized for the musculoskeletal structure using an OFC-like cost function ${ }^{27-29}$. However, it has been suggested that optimal control cannot predict the EMG patterns in wrist movement, despite the fact that the patterns are produced to minimize end-point variance, rather than neural input ${ }^{30}$. On the other hand, some studies have evaluated several cost functions to generate a point-to-manifold reaching task that leaves the target underdetermined ${ }^{31-33}$. Although they suggested the reaching trajectories are generated by the optimality principle with compositions of multiple costs including kinematic smoothness and mechanical energy consumption, it has been remained the question which cost function can predict the EMG patterns in arm movement.

In this study, we re-examined the triphasic muscle activation patterns observed in EMGs during arm movement from the perspective of optimal control using an OFClike cost function that consisted of terminal requirement costs with minimized neural input to predict the muscle activation patterns. Then we performed numerical simulations applying an iterative linear-quadratic-Gaussian (ILQG) method ${ }^{34}$, which approximates OFC, to physiological arm dynamics with a realistic muscle model. This showed that the optimal control could selectively tune muscles in accordance with the movement direction and indicated an interlaced cost based on combinations of the terminal requirement of the end-point position, velocity and force under minimization of neural input during movement. The control induced triphasic muscle activation patterns like those in the recorded EMGs, only under certain conditions validating the position, velocity and force costs. Thus each cost corresponded to each burst of the agonist and antagonist muscles (i.e. AG1, ANT and AG2). Consequently, we suggest that the neural system controls the body by using an optimal control mechanism based 
on a cost function that consists of position, velocity and force requirements, and that they appear to correspond to the first (AG1), second (ANT) and third (AG2) muscle activation bursts, which serve to initiate, decelerate and stabilize the movement, respectively.

\section{Results}

We simulated movement using an approximate $\mathrm{OFC}^{34}$ and a two-joint six-muscle arm model ${ }^{17,35,36}$ (i.e. shoulder flexor (SF), shoulder extensor (SX), elbow flexor (EF), elbow extensor (EX), biarticular flexor (BF) and biarticular extensor (BX) muscles). We set the simulation duration to $500 \mathrm{~ms}$ and determined the terminal time of the movement at $400 \mathrm{~ms}$ to fit the experimental data ${ }^{3,17,37}$. Then we assumed four situations involving the various cost weights in equation (9) as Cases 1-4. In Case 1, the cost weights were zero, except the positional cost. The movement was constrained only in the terminal position. In Case 2, the cost weight of the end-point force was zero. This required movement under kinematic constraints. In Case 3, the cost weight of the end-point velocity was zero to inhibit the end-point force at the target position. Finally, Case 4 required the regulation of the end-point position, velocity and force states at the movement end. The task required stopping at the target without force generation. They showed that the hand pathways varied slightly, forming curves or almost-straight lines in all cases in accordance with the direction (Figure 2, left column). 
a

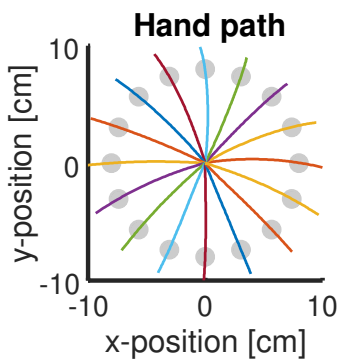

b

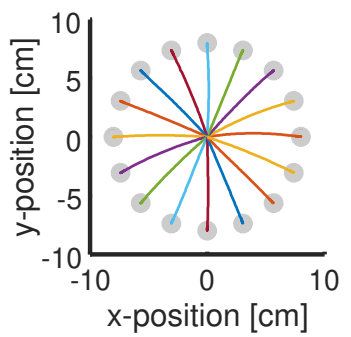

C
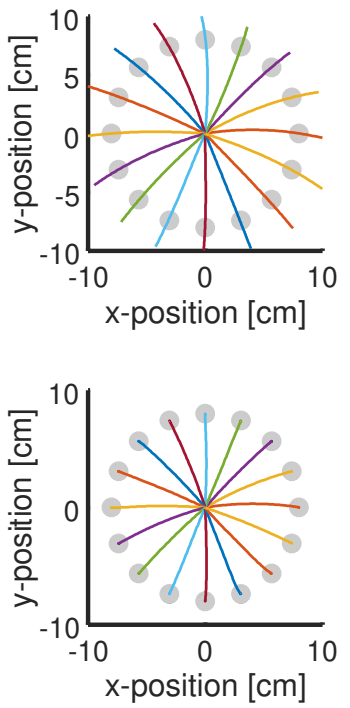
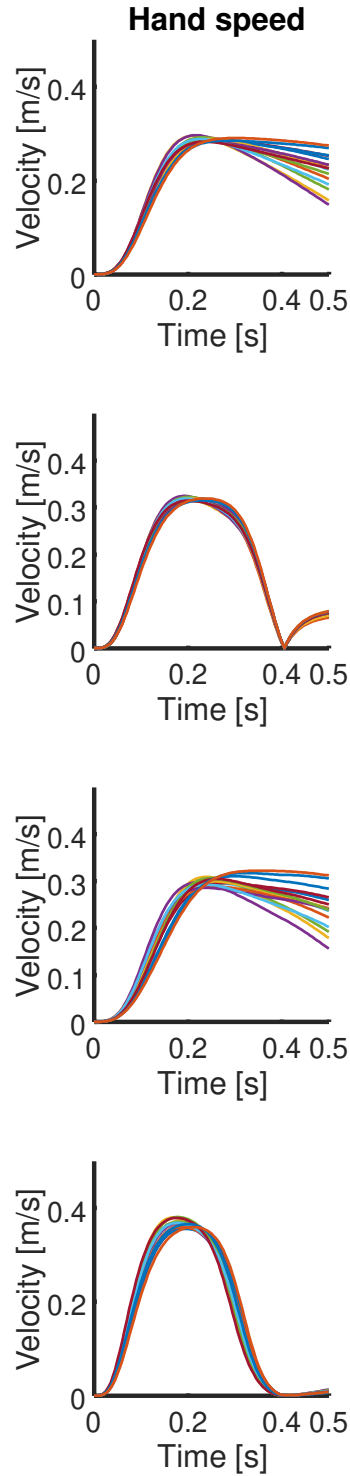
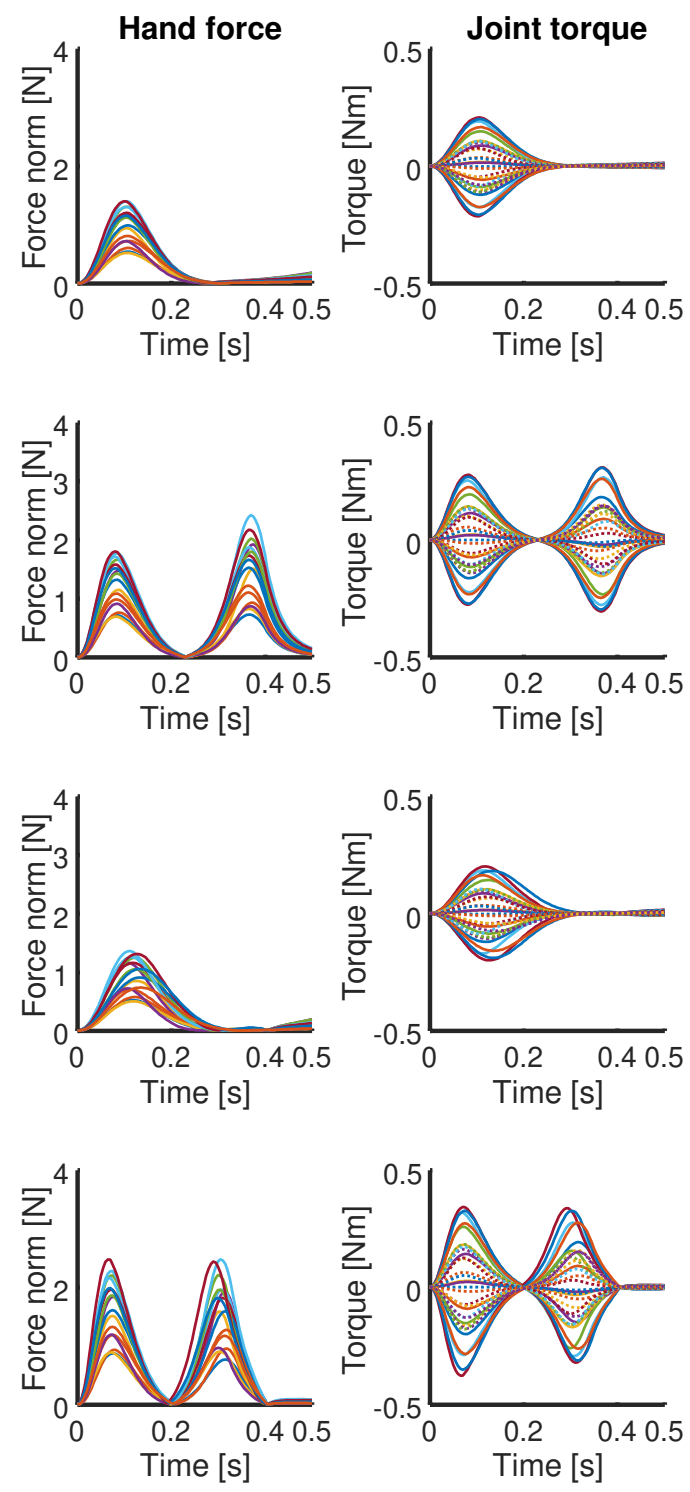

Figure 2. The centre-out movement for 16 targets. The columns from left to right are the hand paths, hand speed profiles, root mean squared hand force profiles and joint torque profiles. In the hand paths, the target positions are shown as filled circles. In the joint torque profiles, solid and dotted lines indicate the torques of the shoulder and elbow joints, respectively. Cases $1-4$ are in rows (a)-(d), respectively. 


\section{Movement parameters}

In Case 1, the hand passed through on the targets and the speed did not converge on zero at the time of the movement end (i.e. $400 \mathrm{~ms}$ ); the joint torques peaked at the onset of movement to generate the hand force triggering the movement (Figure 2a). Then the joint torques and hand force diverged slightly at the movement end because a passive tensile force was generated by stretching the muscles. In Case 2, the hand stopped on the targets and the speed was almost set to zero at the movement end, although they had slight motions after the movement end (Figure $2 b$ ). The hand force and joint torques showed biphasic peaks. The second peak of the joint torques was generated in the direction opposite the movement, to decelerate the movement and set the hand speed to zero at the movement end. However, the hand force and joint torques did not converge on zero at the movement end but gradually decreased after then. In Case 3, the hand passed through on the targets and the speed was not zero at the movement end time, similar to Case 1, and the hand force and joint torques converged on zero at this time in contrast to Case 1 (Figure 2c). In Case 4, the hand stopped on the targets and the speed was close to zero with gradual curves and formed clear bell-shaped profiles (Figure 2d). Although the hand force and joint torques showed biphasic peaks similar to Case 2, they converged on zero at the movement end.

\section{Muscle activation patterns}

Although the muscles were activated selectively in accordance with the movement direction, regardless of the case following the first muscle activation (AG1), the second (ANT) and third (AG2) bursts of the muscles varied temporally among the cases (Figure 3). 

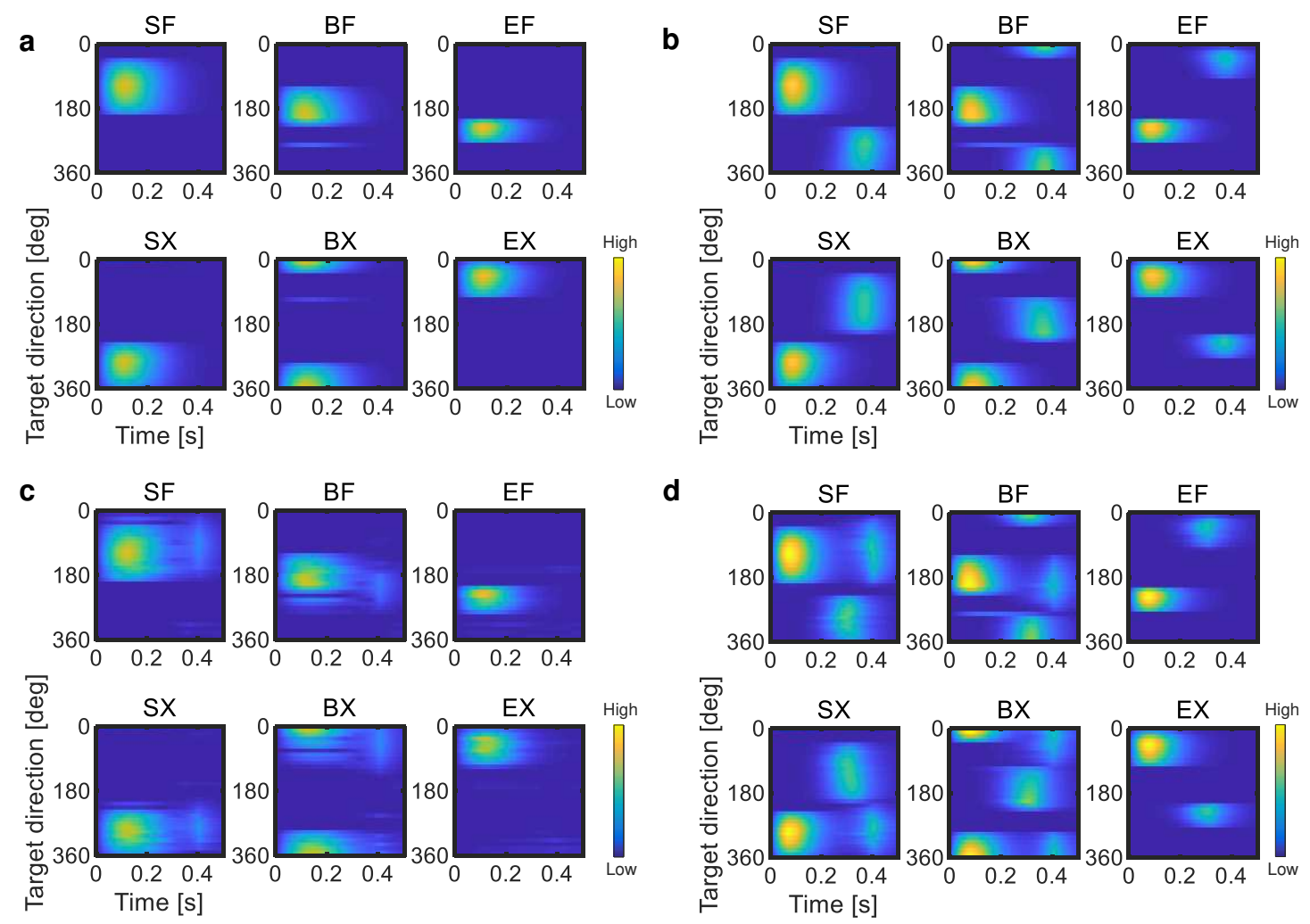

\section{Figure 3. Muscle activation patterns plotted as a function of time and target}

direction. Cases 1-4 are shown in (a)-(d), respectively. The values were normalized using the highest activation level in each muscle across all cases.

In Case 1, agonist muscles were activated once to initiate the movement (Figure 3a). Then the monoarticular elbow muscles EF and EX showed no activation. In Case 2, the activation of the agonist and antagonist muscles alternated like a burst of AG1 and ANT, occurring immediately after the beginning and at the end of the movement, respectively (Figure 3b). The antagonist muscles might contribute the torque in the direction opposite to that of the movement to decelerate the movement. However, EF and EX were activated only as the antagonist muscles synchronizing with the biarticular muscles BF or BX. In Case 3, the agonist muscles were activated twice, like bursts of AG1 and AG2 (Figure 3c). The second activations might suppress the development of 
the hand force and joint torques at the end of the movement. Then muscles EF and EX had weak activation to supplement BF or BX. In Case 4, the muscle activations showed a triphasic pattern resembling the superposition of those in Cases 2 and 3, with the agonist and antagonist muscles activated alternately, although the agonist muscles were occasionally activated twice (Figure 3d). Of note, when the SF muscle acted as an agonist muscle (e.g. $90^{\circ}$ movement direction), it was activated twice, at the start and end of the movement, while it was activated only once in the middle of the movement when it was an antagonist muscle (e.g. $270^{\circ}$ movement direction). The later activation of agonist muscles was thought to contribute to stabilization of the movement because the later torque observed in Case 2, which the antagonist muscles generated, may induce unnecessary movement after reaching the target. Thus, the additional torque generated by the agonist muscles was required to counteract the opposite torques and stabilize the movement after completing the task.

\section{Stabilization control}

We suggested that the force cost reactivates the agonist muscle to counteract the breaking torques, for stabilization. An alternate hypothesis is that to achieve a reaching movement in a certain time, it is necessary to optimize the stability of the terminal kinematic state (i.e. position, or position and velocity) after the movement phase but not to minimize only the terminal cost at the movement end. To examine this hypothesis, we define the extra two cost functions for stabilization control as equation (11).

As a result, although the cost function in equation (11) showed the biphasic muscle pattern seen in Case 2 (Figure 4c), the cost function in equation (12) reproduced the triphasic muscle pattern seen in Case 4 (Figure 4d). Accordingly, we could not reject 
the hypothesis of the stabilization control represented by equation (12). However, the stabilization control may regulate a differential of the cost terms, because the stabilization of equation (11), which adopts only the position cost, decreased the hand speed similar to Case 2 (Figure 4a). Therefore we postulate the stabilization control of equation (12) suppressed hand acceleration during the stabilization period (Figure 4c), and it played a role like the force cost in Case 4.
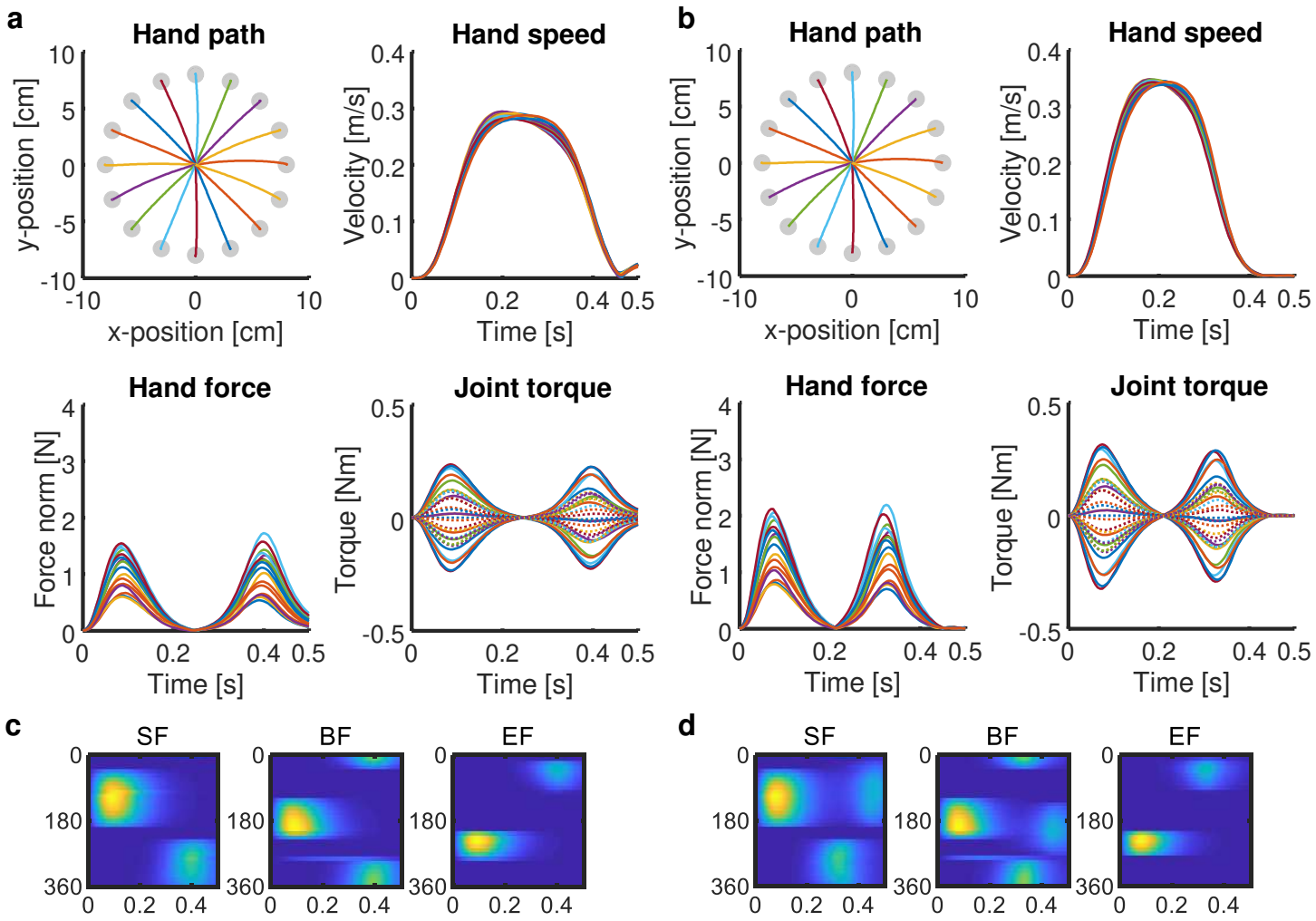

d
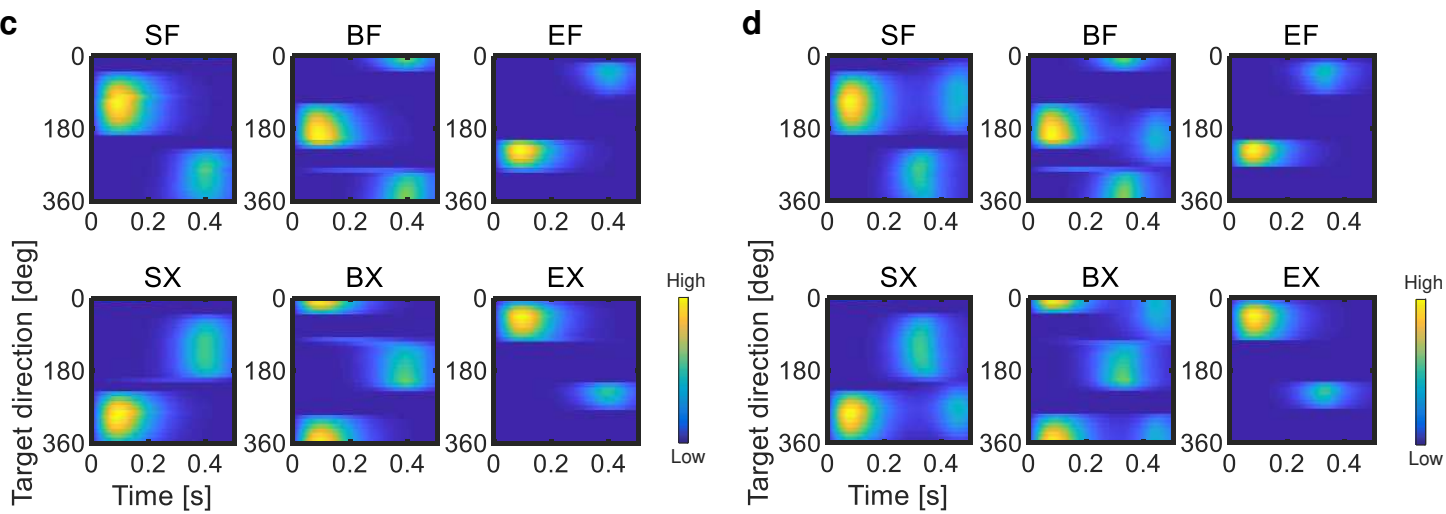

Figure 4. Stabilization control. The controls of only the position cost, and a pair of the position and velocity costs are shown in (a, c) and (b, d), respectively. (a, b) The centre- 
out movement for 16 targets. The format is the same as in Figure 2. (c, d) Muscle activation pattern. The format is the same as in Figure 3.

\section{Discussion}

The purpose of this study was to investigate the requirements for deriving muscle activation patterns in which antagonistic muscle pairs show alternate bursts with triphasic shapes. To examine it, we carried out simulations of arm movement to apply four types of cost function that considered the end-point position, velocity and force requirements under minimization of the neural input. To summarize the results, the position, velocity and force costs were postulated to play following roles: (i) the position cost led to activation of the first agonist muscle, to trigger movement; (ii) the velocity cost activated the antagonist muscles and generated braking torques to decelerate the movement; and (iii) the force cost reactivated the agonist muscle to negate the breaking torques and the passive tensile force of the stretched muscles, for stabilization.

Our control methodology was not truly optimal, but approximately optimal, since solving the optimal control problem analytically for nonlinear systems such as the musculoskeletal system is difficult. According to the original OFC model for a linear dynamics plant whose state variable $\mathbf{x}(t)$ comprises the position, velocity and force ${ }^{14}$, the motor command is represented as $\cdot \mathbf{u}(t)=\mathbf{K}(t) \cdot \hat{\mathbf{x}}(t)$, where $\mathbf{K}(t)$ is the feedback gain and $\hat{\mathbf{x}}(t)$ is the estimated state integrating the prediction of the internal model with the sensory feedback. The components of the feedback gain for the position, velocity and force peak at varied timings according to different cost functions corresponding to 
Cases 1-4 (Figure 5). They show a peak at simultaneous timing in Case 1, twice peaks in Case 2 and Case 3, and the position, velocity and force peak in turn in the early, mid and late phases of the movement, respectively. Thus the position, velocity and force feedback gains serve to initiate, decelerate and stabilize the movement, respectively, which fits our suggestion regarding the roles of the cost terms.
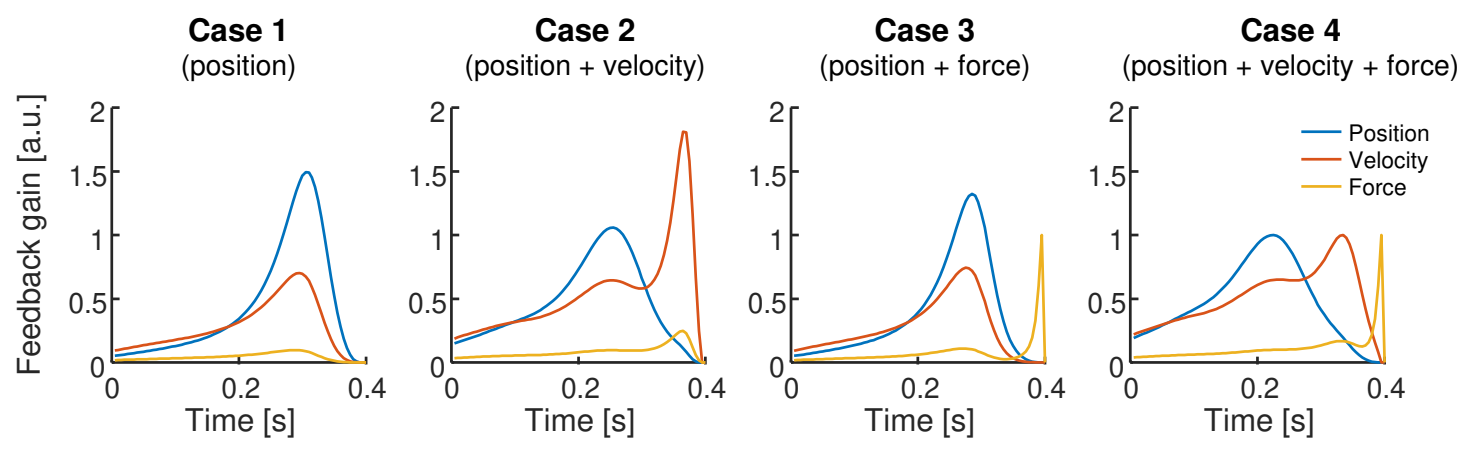

Figure 5. Typical feedback gains of the optimal feedback control (OFC) model for

a linear system. Each of Case 1-4 corresponds to the same formation of the cost function above results. Each value of the position, velocity and force gains is normalised by that of Case 4 .

It seems that the force cost can be replaced with an acceleration term. Although the acceleration term actually could reproduce the triphasic muscle pattern shown in Case 4, it does not seem natural to use the acceleration cost instead of the force. The muscle spindles detect the muscle length and velocity, and the Golgi tendon organ provides the muscle tension. However, the acceleration cannot be gained directly from proprioceptive systems. A previous study reported producing the same muscle pattern to solve an optimal control problem of muscles with boundary conditions, which velocities 
and forces were zero at the beginning and end of the movement ${ }^{38}$. Thus we postulate that the neural system adopts not the acceleration, but the force.

Proprioceptive feedback is not essential to generate the triphasic pattern ${ }^{6}$, although it contributes to achieving precise movements. Thus the triphasic pattern of muscles might be programed in the motor planning phase. In the OFC model, the neural system is also assumed to compute the feedback gain before movement onset, and execute the feedback control for estimated states. The common point implies that the muscle activation pattern serves as feedback gain reflecting muscle properties such as stiffness and viscosity.

Several studies in patients with motor disorders have reported that the basal ganglia may have a role in scaling the size of AG1, reinforcing the voluntary command and inhibiting inappropriate EMG activity ${ }^{6}$. Therefore we assume that in the basal ganglia module, the positional cost weight $w_{p}$ is related to AG1. The cerebrum may regulate the other cost weights $w_{v}$ and $w_{f}$ to balance $w_{p}$, because the cerebellum has a role in timing the voluntary bursts of ANT and AG2. Indeed, cerebellar patients are unable to perform accurate movements ${ }^{39}$. This deficit is known as dysmetria, which is a lack of coordination of movement that results in overshooting or undershooting a target during reaching tasks.

In previous studies that used similar muscle models ${ }^{28,34}$, the tensile force of the passive elastic component was excluded from the muscle model (i.e. FPE2 in equation (8)), although the force is not small. We assume that this was done because of the sensitivity of parameter selection for the muscle model, such as the moment arms, optimal muscle lengths and optimal joint angles, which affect the muscle length and generate a tensile force without muscle activation. In the model, the tensile force 
requires initial muscle activation to maintain the initial position before movement onset, and this affects the hand trajectories, causing marked distortion. Thus we set the initial hand position to an equilibrium point balancing the forces, to set the initial muscle activations to zero. With such sensitive effects, how do we generate and control movement? Previous studies suggested that the neurons are optimized for physical mechanics $19,27,28$. However, if subjects learned fine motor tasks once, such learning tend to persist, even when clearly suboptimal ${ }^{40}$. Subsequently, the subjects learn to overcome real and virtual changes in their biomechanics, but prefer rescaling their prior motor habits to recomputing to optimize the control policy ${ }^{41}$. The prior motor habits may be learned from muscle activation patterns generated by lower sensorimotor circuitry that is functionally suboptimal. However, we ignored the lower sensorimotor circuitry, which is a limitation of our model. Recently, a model of the spinal circuitry and musculoskeletal system was developed ${ }^{42}$, and seems to be useful for studying habitual motor control systems.

Dehghani and Bahrami suggested that arm movements are planned with some principle patterns of muscle synergies, and the plans are divided into few phases to reduce the dimension of the control space ${ }^{43,44}$. Also, Sakaguchi et al. have proposed a computational model that brain adaptively divides the continuous-time axis into discrete segments and executes feedforward control in each segment to allow sensorimotor delays ${ }^{45}$. However, the OFC could realize the control of the muscle synergies through feedback gains, and the segmentation of motor execution may be identified as some steady-state feedback gains computed by a model predictive control under the framework of the OFC ${ }^{19}$. 
In summary, the results of this study imply that a triphasic muscle activation pattern can be predicted by an optimal control mechanism, e.g. by adopting an OFC-like cost function. Furthermore, the costs of position, velocity and force requirements may be the critical parameters for the physiological control of movements and correspond to the triggering, braking, and stabilizing of movement, respectively.

\section{Methods}

We simulated arm movements using the ILQG method ${ }^{34}$ together with a two-joint sixmuscle arm model ${ }^{17,35,36}$. The ILQG method constructs an affine feedback control law by minimizing a quadratic approximation to optimize a cost function. The muscles were modelled by nonlinear dynamics reflecting the force-length and force-velocity relationships of mammalian skeletal muscle ${ }^{46}$. We examined a centre-out reaching movement task in this study. The hand position at the start of movement was located 22 $\mathrm{cm}$ in front and $8 \mathrm{~cm}$ to the right of the shoulder, which is nearly equal to an equilibrium point balanced by passive elastic muscle forces. Then, 16 targets were aligned $8 \mathrm{~cm}$ away from the initial hand position. All of the simulations in this paper used simple Euler integration with a 5-ms time step for $500 \mathrm{~ms}$, and we defined the movement duration as $400 \mathrm{~ms}$ according to the experimental measurement in monkeys ${ }^{3,17,37}$.

\section{Musculoskeletal arm model}

We considered the monkey's arm to be a two-joint arm composed of the shoulder and elbow joints. The joint angles were defined as vector $\boldsymbol{\theta}=\left[\theta_{1}, \theta_{2}\right]^{T}$, where $\theta_{1}$ and $\theta_{2}$ 
indicate the shoulder and elbow variables, respectively. Suppose joint torque $\tau \in R^{2}$, the dynamics of the monkey's arm in horizontal plane are denoted by

$$
\tau=\mathbf{M}(\boldsymbol{\theta}) \cdot \mathbf{C}(\boldsymbol{\theta}, \boldsymbol{\theta})+\mathbf{D} \cdot \boldsymbol{\theta},
$$

where $\mathbf{M}(\cdot) \in R^{2 \times 2}, \mathbf{C}(\cdot) \in R^{2}$, and $\mathbf{D} \in R^{2 \times 2}$ are the inertia matrix, Coriolis force vector, and viscosity matrix, respectively, and are given by

$$
\begin{gathered}
\mathbf{M}(\boldsymbol{\theta})=\left[\begin{array}{cc}
s_{1}+2 s_{2} \cos \theta_{2} & s_{3}+s_{2} \cos \theta_{2} \\
s_{3}+s_{2} \cos \theta_{2} & s_{3}
\end{array}\right], \\
\mathbf{C}(\boldsymbol{\theta}, \boldsymbol{\theta})=\left[\begin{array}{c}
-\&_{2}\left(2 \theta_{1}^{\alpha_{1}}+\theta_{2}\right) \\
\delta_{1}^{2}
\end{array}\right] \cdot s_{2} \sin \theta_{2}, \mathbf{D}=\left[\begin{array}{ll}
d_{11} & d_{12} \\
d_{21} & d_{22}
\end{array}\right], \\
s_{1}=I_{1}+I_{2}+m_{2} l_{1}^{2}, s_{2}=m_{2} l_{1} l_{g 2}, s_{3}=I_{2} .
\end{gathered}
$$

They are represented by the link parameters: i.e. mass $m_{i}$, length $l$, distance from the joint centre of mass $l_{g i}$, moment of inertia $I_{i}$, joint friction $d_{i l}, d_{i 2}(i=1$, upper arm; $i=2$, forearm). The parameters are shown in Table 1, and were estimated from our measurements of a Japanese macaque. Because many muscles act on the arm in the horizontal plane, we modelled only two degrees of freedom actuated by six muscle groups: SF, shoulder flexor; SX, shoulder extensor; BF, biarticulate flexor; BX, biarticulate extensor; EF, elbow flexor; and EX, elbow extensor (Figure 6a). The joint torque is a function of its moment arms $\mathbf{A} \in R^{2 \times 6}$ and the muscle tension vector $\mathbf{T}=\left[T_{1}\right.$, $\left.T_{2}, \cdots, T_{6}\right]^{T}$, and it is given by $\boldsymbol{\tau}=\mathbf{A} \cdot \mathbf{T}$. The moment arm is defined as the perpendicular distance from the muscle line of action to the joint centre of rotation, given by

$$
\mathbf{A}=\left[\begin{array}{cccccc}
1.5 & -1.5 & 0 & 0 & 1.5 & -1.5 \\
0 & 0 & 1.5 & -1.5 & 1.5 & -1.5
\end{array}\right] / 100
$$

The $j^{\text {th }}$ muscle activation $a_{j}(j=1,2, \cdots, 6)$ is not equal to the instantaneous neural input $u_{j}$, but is generated by passing $u_{j}$ thorough a filter that describes the calcium dynamics modelled with a first-order nonlinear filter ${ }^{47}$ : 


$$
\underset{j}{\&}=\frac{u_{j}-a_{j}}{f\left(u_{j}, a_{j}\right)},
$$

where

$$
f\left(u_{j}, a_{j}\right)=\left\{\begin{array}{cc}
t_{\text {deact }}+u_{j}\left(t_{\text {act }}-t_{\text {deact }}\right) & u_{j}>a_{j} \\
t_{\text {deact }} & u_{j} \leq a_{j}
\end{array} .\right.
$$

The time constant parameters were set as $t_{\text {act }}=0.05[\mathrm{~s}]$ and $t_{\text {deacct }}=0.066[\mathrm{~s}]$ because the input-dependent activation dynamics are faster than the constant deactivation dynamics (Figure 6b).
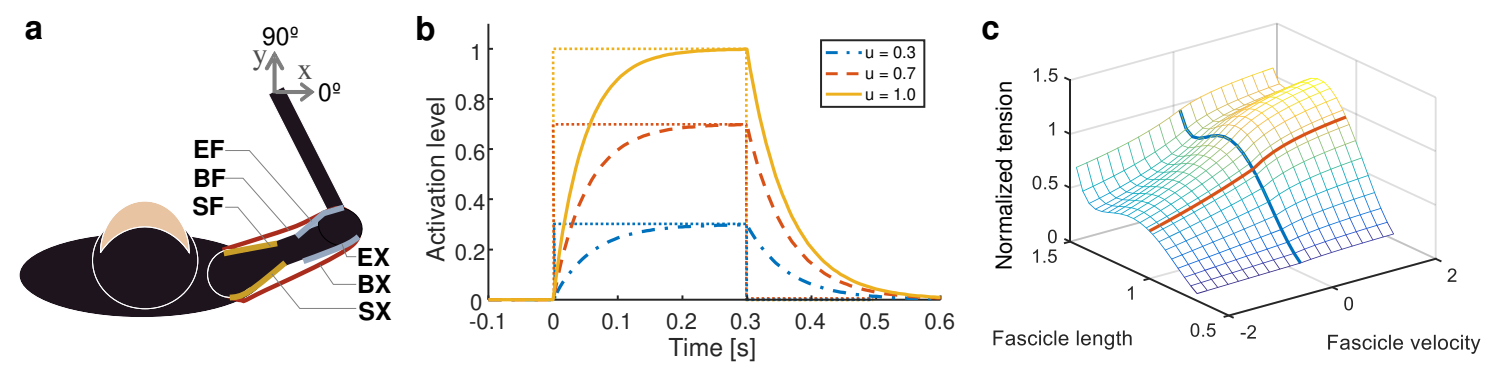

Figure 6. Simulation model. (a) Allocation of muscles in the two-link, six-muscle arm model. The SF models the pectoralis major, coracobrachialis and deltoid anterior muscles. The SX models the posterior and middle deltoid muscles. The BF models the long and short biceps muscles. The BX models the long triceps muscle. The elbow flexor (EF) models the brachialis, brachioradialis and extensor carpi radialis longus muscles. The elbow extensor (EX) models the lateral and medial triceps muscles. (b) Muscle activation dynamics. (c) Length-velocity-tension curve of the muscle model. 
Table 1. Link parameters.

\begin{tabular}{ccccccc}
\hline \hline Link & $\begin{array}{c}m_{i} \\
{[\mathrm{~kg}]}\end{array}$ & $\begin{array}{c}l_{i} \\
{[\mathrm{~m}]}\end{array}$ & $\begin{array}{c}l_{g i} \\
{[\mathrm{~m}]}\end{array}$ & $\begin{array}{c}I_{i} \\
{\left[\mathrm{~kg} \cdot \mathrm{m}^{2}\right]}\end{array}$ & $\begin{array}{c}d_{i 1} \\
{[\mathrm{~N} \cdot \mathrm{m} \cdot \mathrm{s} / \mathrm{rad}]}\end{array}$ \\
\hline$i=1$ & 0.3 & 0.15 & 0.07 & $5.0 \times 10^{-3}$ & $5.0 \times 10^{-3}$ & $2.5 \times 10^{-3}$ \\
$i=2$ & 0.3 & 0.21 & 0.12 & $9.0 \times 10^{-3}$ & $2.5 \times 10^{-3}$ & $5.0 \times 10^{-3}$ \\
\hline \hline
\end{tabular}

Mammalian muscles have remarkable scaling properties, meaning that all are similar after proper normalization: length is expressed in units of the length at which the maximum isometric force is generated ${ }^{48}$, and velocity is expressed in the units per second ${ }^{46}$. Thus we assume the units are unified across all muscles as $L^{0}=0.08[\mathrm{~m}]$, and denote a normalized muscle length $L_{j}$ as follows:

$$
L_{j}=1+A_{j}^{T}\left(\boldsymbol{\theta}_{j}^{0}-\boldsymbol{\theta}\right) / L^{0},
$$

where $A_{j}$ is the $j^{\text {th }}$ row vector of $\mathbf{A}, \boldsymbol{\theta}_{j}^{0} \in R^{2}$ is the optimal joint angle vector of the $j^{\text {th }}$ muscle for generating the maximal torque in the shoulder and elbow, respectively. Then the muscle tension $T_{j}$ is given to scale the unit-less tension by the absolute muscle force of the physiological cross-sectional area (PCSA) to yield the physical tension ${ }^{49}$ :

$$
T_{j}=F_{a} \cdot P \cdot \bar{T}_{j},
$$

where $F_{a}$ is the absolute muscle force and is set to $F_{a}=32\left[\mathrm{~N} / \mathrm{cm}^{2}\right]$ based on measurements in monkeys ${ }^{50}$, and $P$ is the PCSA which is assumed to unify across the muscles as $P=10\left[\mathrm{~cm}^{2}\right]$. According to a model of mammalian skeletal muscle ${ }^{46,51}$, the unit-less muscle tension $\bar{T}_{j}$ is produced by a nonlinear muscle model composed of the functions of contractile element $F_{C E}(\cdot)$ and passive elastic element $F_{P E}(\cdot)$ :

$$
\bar{T}_{j}=F_{C E}\left(a_{j}, L_{j}, E_{j}^{\&}\right)+F_{P E}\left(L_{j}\right),
$$

where 


$$
\begin{aligned}
& F_{C E}\left(a_{j}, L_{j}, \mathbb{L}_{j}^{\&}\right)=A_{f}\left(a_{j}, L_{j}\right) \cdot F_{L}\left(L_{j}\right) \cdot F_{V}\left(L_{j}, L_{j}^{\&}\right), \\
& F_{P E}\left(a_{j}, L_{j}\right)=F_{P E 1}\left(L_{j}\right)+A_{f}\left(a_{j}, L_{j}\right) \cdot F_{P E 2}\left(L_{j}\right) .
\end{aligned}
$$

Here $A_{f}(\cdot), F_{L}(\cdot), F_{V}(\cdot)$ are the functions of the activation-frequency relationship, the tetanic force-length relationship, and the tetanic force-velocity relationship, respectively. The passive elastic force is represented by two separate functions, $F_{P E 1}(\cdot)$ and $F_{P E 2}(\cdot)$, which exert a tensile force and resists compression force, respectively. They are defined as follows:

$$
\begin{gathered}
A_{f}\left(a_{j}, L_{j}\right)=1-\exp \left[-\left\{\frac{a_{j}}{0.56\left(2.12+3.31\left(1 / L_{j}-1\right)\right)}\right\}^{2.12+3.31\left(1 / L_{j}-1\right)}\right], \\
F_{L}\left(L_{j}\right)=\exp \left(-\mid \frac{L_{j}^{1.55}-\left.1\right|^{2.12}}{0.81}\right), \\
F_{V}\left(L_{j}, E_{j}^{\&}\right)=\left\{\begin{array}{c}
\frac{-7.39-E_{j}^{\&}}{-7.39-\left(3.21-4.17 L_{j}\right) E_{j}^{\&}} \quad E_{j}^{\&} \leq 0 \\
F_{P E 1}\left(L_{j}\right)=0.05+1.53 E_{j}^{\&} \\
F_{P E 2}\left(L_{j}\right)=\min \left[-0.02\left\{\log \left(18.7\left(L_{j}-0.79\right)\right)-1\right\}, 0\right] .
\end{array}\right.
\end{gathered}
$$

The dependence of force on the length and velocity of a muscle is often referred to as the force-length and force-velocity curves, respectively (Figure 6c). The muscle model parameters were shown in Table 2, which are assigned based on the results of anatomical measurements of macaque monkeys ${ }^{50,52}$. 
Table 2. Equilibrium joint angles of each muscle. *does not affect the muscle length regardless of the value, because the moment arms are set to zero so as not to transform the effects.

\begin{tabular}{|c|c|c|c|c|c|c|c|}
\hline & & $j=1$ & $j=2$ & $j=3$ & $j=4$ & $j=5$ & $j=6$ \\
\hline \multirow{2}{*}{$\frac{180}{\pi} \boldsymbol{\theta}_{j}^{0}$} & & 15 & 5 & -* & -* $^{*}$ & 15 & 5 \\
\hline & & _* & _* & 90 & 110 & 100 & 100 \\
\hline
\end{tabular}

\section{Approximately optimal feedback control}

We transformed the two-joint six-muscle model into a state-space model. The control object was denoted by the state vector $\mathbf{x} \in R^{10}$ as

$$
\mathbf{x}=\left[\begin{array}{l}
\boldsymbol{\theta} \\
\boldsymbol{\theta} \\
\mathbf{a}
\end{array}\right],
$$

where $\mathbf{a}=\left[a_{1}, a_{2}, \ldots, a_{6}\right]^{T}$ is the muscle activation vector. Here we define the state vector at time $t$ as $\mathbf{x}(t)$. Then the dynamics of the musculoskeletal arm model can be written as a state-space equation described as

$$
(t)=F(\mathbf{x}(t))+G(\mathbf{x}(t)) \cdot \mathbf{u}(t) .
$$

The nonlinear functions $F(\cdot)$ and $G(\cdot)$ are defined for descriptive purposes to represent the dynamics in an affine form. In practice, they are given as locally linearized forms around each state at time $t$ to obtain an approximately OFC law. The motor command $\mathbf{u}(t)$ is computed iteratively using the Levenberg-Marquardt algorithm to optimize the following cost function:

$$
q_{1}=\min _{\mathbf{u}}\left(w_{p}\left\|\mathbf{p}\left(T_{s}\right)-\mathbf{p}^{*}\right\|^{2}+w_{v}\left\|\mathbf{v}\left(T_{s}\right)\right\|^{2}+w_{f}\left\|\mathbf{f}\left(T_{s}\right)\right\|^{2}+\int_{0}^{T}\|\mathbf{u}(t)\|^{2} d t\right),
$$


where $T_{s}$ and $T$ are the terminal time of the movement and simulation duration, respectively (i.e. $T_{s}=0.4[\mathrm{~s}]$ and $\left.T=0.5[\mathrm{~s}]\right)$. The vectors $\mathbf{p}\left(T_{s}\right) \in R^{2}, \mathbf{v}\left(T_{s}\right) \in R^{2}$ and $\mathbf{f}\left(T_{s}\right) \in R^{2}$ are the end-point position, velocity and force in Cartesian space at the movement end $T_{s}$, respectively. They are calculated from the joint angles, angular velocities and torques as follows:

$$
\begin{gathered}
\mathbf{p}(t)=\left[\begin{array}{c}
l_{1} \cos \theta_{1}(t)+l_{2} \cos \left(\theta_{1}(t)+\theta_{2}(t)\right) \\
l_{1} \sin \theta_{1}(t)+l_{2} \sin \left(\theta_{1}(t)+\theta_{2}(t)\right)
\end{array}\right], \mathbf{v}(t)=\mathbf{J}(t) \cdot \nLeftarrow(t), \\
\mathbf{f}(t)=\left(\mathbf{J}(t)^{T}\right)^{-1} \cdot \boldsymbol{\tau}(t),
\end{gathered}
$$

where $\mathbf{J}(t) \in R^{2 \times 2}$ is the Jacobian matrix

$$
\mathbf{J}(t)=\left[\begin{array}{cc}
-l_{1} \sin \theta_{1}(t)-l_{2} \sin \left(\theta_{1}(t)+\theta_{2}(t)\right) & -l_{2} \sin \left(\theta_{1}(t)+\theta_{2}(t)\right) \\
l_{1} \cos \theta_{1}(t)+l_{2} \cos \left(\theta_{1}(t)+\theta_{2}(t)\right) & l_{2} \cos \left(\theta_{1}(t)+\theta_{2}(t)\right)
\end{array}\right] .
$$

In addition, $\mathbf{p}^{*}$ is a target position in Cartesian space, and $w_{p}, w_{v}$ and $w_{f}$ are cost weights of the position, velocity and force requirements at the movement end, respectively. On the right-hand side of equation (10), the first, second and third terms evaluate the requirements of the end-point position, velocity and force, respectively, at the end of movement in achieving the desired state, whereby the end-point is close to the target position with zero values for the end-point velocity and force. The fourth term, which is the sum of the squares of the neural inputs during the movement, evaluates the metabolic cost of the neural input.

\section{Cost weight parameters}

We simulated four situations requiring movement under minimized neural input by balancing the cost weights in equation (10) (Table 3). These were determined heuristically to achieve the task. We confirmed that the results in this study are robust to 
changes in the parameters according to the sensitivity analysis (see Supplementary Note).

Table 3. Cost weight parameters.

\begin{tabular}{lccc}
\hline \hline & $w_{p}$ (position) & $w_{v}$ (velocity) & $w_{f}$ (force) \\
\hline Case 1 & 1000 & 0 & 0 \\
Case 2 & 1000 & 100 & 0 \\
Case 3 & 1000 & 0 & 10 \\
Case 4 & 1000 & 100 & 10 \\
\hline \hline
\end{tabular}

\section{Stabilization control}

We examined an alternate hypothesis that stabilizes the terminal position, or position and velocity after the movement phase but not to minimize only the terminal cost at the movement end. Thus, we additionally defined following two cost functions for the stabilization control:

$$
q_{2}=\min _{\mathbf{u}}\left[\frac{1}{T-T_{s}} \int_{T_{s}}^{T} w_{p}\left\|\mathbf{p}(t)-\mathbf{p}^{*}\right\|^{2} d t+\int_{0}^{T}\|\mathbf{u}(t)\|^{2} d t\right],
$$

and

$$
q_{3}=\min _{\mathbf{u}}\left[\frac{1}{T-T_{s}} \int_{T_{s}}^{T}\left(w_{p}\left\|\mathbf{p}(t)-\mathbf{p}^{*}\right\|^{2}+w_{v}\|\mathbf{v}(t)\|^{2}\right) d t+\int_{0}^{T}\|\mathbf{u}(t)\|^{2} d t\right] .
$$

Then, we set the time at the movement end and stabilization period as $0.5 \mathrm{~s}$ and $0.1 \mathrm{~s}$, respectively (i.e. $T_{s}=0.4[\mathrm{~s}], T=0.5[\mathrm{~s}]$ ). 


\section{Acknowledgements}

A part of this work was supported by JSPS KAKENHI Grant Numbers JP25880031, JP26702023 and JP19K20745.

\section{Author contributions}

All of this study was conducted by Y.U.

\section{Competing interests}

The authors declare no competing interests.

\section{Data availability}

The MATLAB (MathWorks, Natick, MA, USA) codes and data sets that support the findings of this study are available in Github (https://github.com/yuki-ueyama/MuscleActivation-Pattern).

\section{References}

1 Darainy, M. \& Ostry, D. J. Muscle cocontraction following dynamics learning. Exp. Brain Res. 190, 153-163, doi:10.1007/s00221-008-1457-y (2008).

2 Hallett, M., Shahani, B. T. \& Young, R. R. EMG analysis of stereotyped voluntary movements in man. Journal of Neurology, Neurosurgery, and Psychiatry 38, 1154-1162 (1975).

3 Ueyama, Y. \& Miyashita, E. Devising a Robotic Arm Manipulandum for Normal and Altered Reaching Movements to Investigate Brain Mechanisms of Motor Control. Instrum. Sci. Technol. 41, 251-273, doi:10.1080/10739149.2012.749492 (2013).

4 Kumamoto, M., Oshima, T. \& Yamamoto, T. Control properties induced by the existence of antagonistic pairs of bi-articular muscles - Mechanical engineering model analyses. Hum. Movement Sci. 13, 611-634, doi:10.1016/0167-9457(94)90009-4 (1994).

5 Cooke, J. D. \& Brown, S. H. Movement-related phasic muscle activation. II. Generation and functional role of the triphasic pattern. J. Neurophysiol. 63, 465472, doi:10.1152/jn.1990.63.3.465 (1990). 
6 Berardelli, A. et al. Single-joint rapid arm movements in normal subjects and in patients with motor disorders. Brain 119, 661-674, doi:DOI

10.1093/brain/119.2.661 (1996).

7 Brown, S. H. \& Cooke, J. D. Movement-related phasic muscle activation. I. Relations with temporal profile of movement. J. Neurophysiol. 63, 455-464, doi:10.1152/jn.1990.63.3.455 (1990).

8 Cooke, J. D. \& Brown, S. H. Movement-related phasic muscle activation. III. The duration of phasic agonist activity initiating movement. Exp. Brain Res. 99, 473-482 (1994).

9 Sergio, L. E., Hamel-Paquet, C. \& Kalaska, J. F. Motor cortex neural correlates of output kinematics and kinetics during isometric-force and arm-reaching tasks. J. Neurophysiol. 94, 2353-2378, doi:10.1152/jn.00989.2004 (2005).

10 Trainin, E., Meir, R. \& Karniel, A. Explaining patterns of neural activity in the primary motor cortex using spinal cord and limb biomechanics models. $J$. Neurophysiol. 97, 3736-3750, doi:10.1152/jn.01064.2006 (2007).

11 Georgopoulos, A. P., Kettner, R. E. \& Schwartz, A. B. Primate motor cortex and free arm movements to visual targets in three-dimensional space. II. Coding of the direction of movement by a neuronal population. J. Neurosci. 8, 2928-2937 (1988).

12 Todorov, E. \& Jordan, M. I. Optimal feedback control as a theory of motor coordination. Nat. Neurosci. 5, 1226-1235, doi:10.1038/nn963 (2002).

13 Todorov, E. Optimality principles in sensorimotor control. Nat. Neurosci. 7, 907-915, doi:10.1038/nn1309 (2004).

14 Todorov, E. Stochastic optimal control and estimation methods adapted to the noise characteristics of the sensorimotor system. Neural Comput. 17, 1084-1108, doi:Doi 10.1162/0899766053491887 (2005).

15 Liu, D. \& Todorov, E. Evidence for the flexible sensorimotor strategies predicted by optimal feedback control. J. Neurosci. 27, 9354-9368, doi:10.1523/Jneurosci.1110-06.2007 (2007).

16 Izawa, J., Rane, T., Donchin, O. \& Shadmehr, R. Motor adaptation as a process of reoptimization. J. Neurosci. 28, 2883-2891, doi:10.1523/Jneurosci.535907.2008 (2008).

17 Ueyama, Y. \& Miyashita, E. Optimal Feedback Control for Predicting Dynamic Stiffness During Arm Movement. IEEE Trans. Ind. Electron. 61, 1044-1052, doi:10.1109/Tie.2013.2273473 (2014).

18 Nagengast, A. J., Braun, D. A. \& Wolpert, D. M. Optimal control predicts human performance on objects with internal degrees of freedom. PLoS Comput. Biol. 5, e1000419, doi:10.1371/journal.pcbi.1000419 (2009).

19 Ueyama, Y. Optimal feedback control to describe multiple representations of primary motor cortex neurons. J. Comput. Neurosci. 43, 93-106, doi:10.1007/s10827-017-0650-z (2017).

20 Mitrovic, D., Klanke, S., Osu, R., Kawato, M. \& Vijayakumar, S. A Computational Model of Limb Impedance Control Based on Principles of Internal Model Uncertainty. Plos One 5, doi:ARTN e13601 10.1371/journal.pone.0013601 (2010).

21 Ueyama, Y. \& Miyashita, E. Signal-Dependent Noise Induces Muscle CoContraction to Achieve Required Movement Accuracy: A Simulation Study with 
an Optimal Control. Curr Bioinform 8, 16-24, doi:10.2174/1574893611308010005 (2013).

22 Flash, T. \& Hogan, N. The coordination of arm movements: an experimentally confirmed mathematical model. J Neurosci 5, 1688-1703 (1985).

23 Uno, Y., Kawato, M. \& Suzuki, R. Formation and control of optimal trajectory in human multijoint arm movement. Minimum torque-change model. Biol.

Cybern. 61, 89-101 (1989).

24 Osu, R. et al. Optimal impedance control for task achievement in the presence of signal-dependent noise. J. Neurophysiol. 92, 1199-1215, doi:10.1152/jn.00519.2003 (2004).

25 Pruszynski, J. A., Kurtzer, I. \& Scott, S. H. Rapid motor responses are appropriately tuned to the metrics of a visuospatial task. J. Neurophysiol. 100, 224-238, doi:10.1152/jn.90262.2008 (2008).

26 Pruszynski, J. A. et al. Primary motor cortex underlies multi-joint integration for fast feedback control. Nature 478, 387-390, doi:10.1038/nature10436 (2011).

27 Hirashima, M. \& Nozaki, D. Learning with slight forgetting optimizes sensorimotor transformation in redundant motor systems. PLoS Comput. Biol. 8, e1002590, doi:10.1371/journal.pcbi.1002590 (2012).

28 Lillicrap, T. P. \& Scott, S. H. Preference distributions of primary motor cortex neurons reflect control solutions optimized for limb biomechanics. Neuron 77, 168-179, doi:10.1016/j.neuron.2012.10.041 (2013).

29 Ueyama, Y. Mini-max feedback control as a computational theory of sensorimotor control in the presence of structural uncertainty. Front. Comput. Neurosci. 8, 119, doi:10.3389/fncom.2014.00119 (2014).

30 Haruno, M. \& Wolpert, D. M. Optimal control of redundant muscles in steptracking wrist movements. J. Neurophysiol. 94, 4244-4255, doi:10.1152/jn.00404.2005 (2005).

31 Berret, B., Chiovetto, E., Nori, F. \& Pozzo, T. Manifold reaching paradigm: how do we handle target redundancy? J. Neurophysiol. 106, 2086-2102, doi:10.1152/jn.01063.2010 (2011).

32 Berret, B., Chiovetto, E., Nori, F. \& Pozzo, T. Evidence for Composite Cost Functions in Arm Movement Planning: An Inverse Optimal Control Approach. PLoS Comput. Biol. 7, e1002183, doi:10.1371/journal.pcbi.1002183 (2011).

33 Wochner, I. et al. Optimality Principles in Human Point-to-Manifold Reaching Accounting for Muscle Dynamics. Front. Comput. Neurosci. 14, doi:10.3389/fncom.2020.00038 (2020).

34 Li, W. \& Todorov, E. Iterative linearization methods for approximately optimal control and estimation of non-linear stochastic system. Int. J. Contr. 80, 14391453, doi:10.1080/00207170701364913 (2007).

35 Izawa, J., Kondo, T. \& Ito, K. Biological arm motion through reinforcement learning. Biol. Cybern. 91, 10-22, doi:10.1007/s00422-004-0485-3 (2004).

36 Kambara, H., Kim, K., Shin, D., Sato, M. \& Koike, Y. Learning and generation of goal-directed arm reaching from scratch. Neural Netw 22, 348-361, doi:S0893-6080(08)00265-7 [pii] 10.1016/j.neunet.2008.11.004 (2009).

37 Ueyama, Y. in 20th International Conference on Neural Information Processing. 241-248 (Springer, 2013). 
38 Guigon, E., Baraduc, P. \& Desmurget, M. Computational Motor Control:

Redundancy and Invariance. J. Neurophysiol. 97, 331-347,

doi:10.1152/jn.00290.2006 (2007).

39 Manto, M. Mechanisms of human cerebellar dysmetria: experimental evidence and current conceptual bases. J. Neuroeng. Rehabil. 6, 10, doi:10.1186/17430003-6-10 (2009).

40 de Rugy, A., Loeb, G. E. \& Carroll, T. J. Muscle Coordination Is Habitual Rather than Optimal. J. Neurosci. 32, 7384-7391, doi:10.1523/Jneurosci.579211.2012 (2012).

41 Loeb, G. E. Optimal isn't good enough. Biol. Cybern. 106, 757-765, doi:10.1007/s00422-012-0514-6 (2012).

42 Tsianos, G. A., Goodner, J. \& Loeb, G. E. Useful properties of spinal circuits for learning and performing planar reaches. J. Neural Eng. 11, doi:Artn 056006 10.1088/1741-2560/11/5/056006 (2014).

43 Dehghani, S. \& Bahrami, F. How does the CNS control arm reaching movements? Introducing a hierarchical nonlinear predictive control organization based on the idea of muscle synergies. PLOS ONE 15, e0228726, doi:10.1371/journal.pone.0228726 (2020).

44 Dehghani, S. \& Bahrami, F. 3D human arm reaching movement planning with principal patterns in successive phases. J. Comput. Neurosci. 48, 265-280, doi:10.1007/s10827-020-00749-2 (2020).

45 Sakaguchi, Y., Tanaka, M. \& Inoue, Y. Adaptive intermittent control: A computational model explaining motor intermittency observed in human behavior. Neural Netw 67, 92-109, doi:https://doi.org/10.1016/j.neunet.2015.03.012 (2015).

46 Brown, I. E., Cheng, E. J. \& Loeb, G. E. Measured and modeled properties of mammalian skeletal muscle. II. The effects of stimulus frequency on forcelength and force-velocity relationships. J. Muscle. Res. Cell. Motil. 20, 627-643, doi:Doi 10.1023/A:1005585030764 (1999).

47 Ashley, C. C. \& Ridgway, E. B. On the relationships between membrane potential, calcium transient and tension in single barnacle muscle fibres. $J$. Physiol. 209, 105-130, doi:10.1113/jphysiol.1970.sp009158 (1970).

48 Brown, I. E., Liinamaa, T. L. \& Loeb, G. E. Relationships between range of motion, $\mathrm{L}(0)$, and passive force in five strap-like muscles of the feline hind limb. J. Morphol. 230, 69-77, doi:Doi 10.1002/(Sici)10974687(199610)230:1<69::Aid-Jmor6>3.0.Co;2-I (1996).

49 Scott, S. H., Brown, I. E. \& Loeb, G. E. Mechanics of feline soleus: I. Effect of fascicle length and velocity on force output. J. Muscle. Res. Cell. Motil. 17, $207-$ 219, doi:10.1007/BF00124243 (1996).

50 Cheng, E. J. \& Scott, S. H. Morphometry of Macaca mulatta forelimb. I. Shoulder and elbow muscles and segment inertial parameters. J. Morphol. 245, 206-224, doi:Doi 10.1002/1097-4687(200009)245:3<206::Aid-Jmor3>3.0.Co;2$\mathrm{U}(2000)$.

51 Tsianos, G. A., Rustin, C. \& Loeb, G. E. Mammalian Muscle Model for Predicting Force and Energetics During Physiological Behaviors. IEEE Trans. Neural. Syst. Rehabil. Eng. 20, 117-133, doi:10.1109/Tnsre.2011.2162851 (2012). 
52 Graham, K. M. \& Scott, S. H. Morphometry of Macaca mulatta forelimb. III. Moment arm of shoulder and elbow muscles. J. Morphol. 255, 301-314, doi:10.1002/jmor.10064 (2003). 
Figures

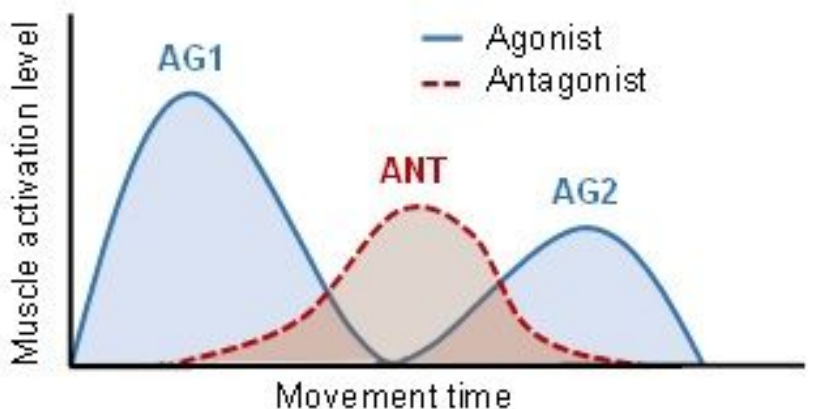

Figure 1

Triphasic muscle activation pattern during reaching movement. AG1, AG2 and ANT indicate the sequence of bursts for the pair of agonist (AG1 and AG2) and antagonist (ANT) muscles.

a

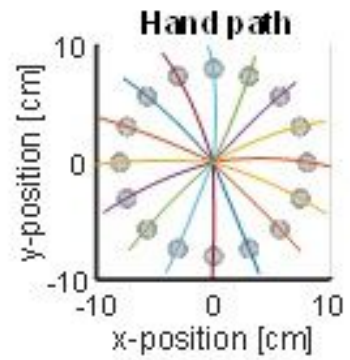

b

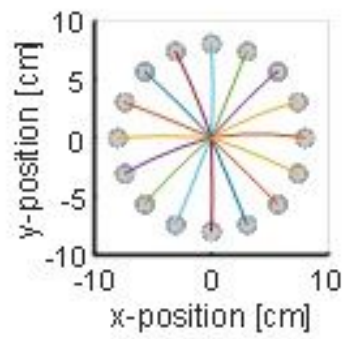

c

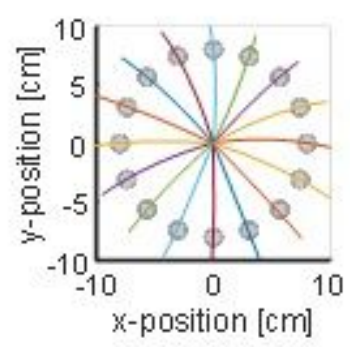

d

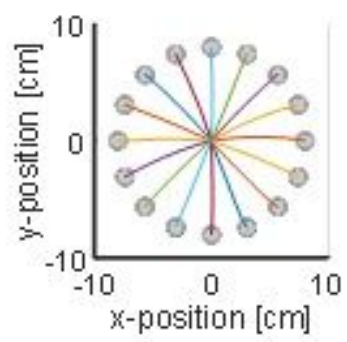

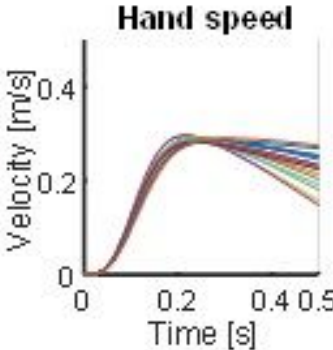
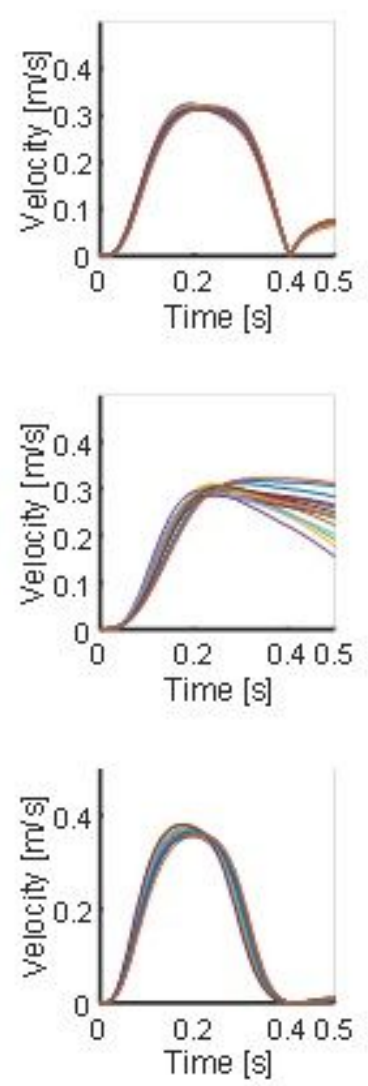

Hand force
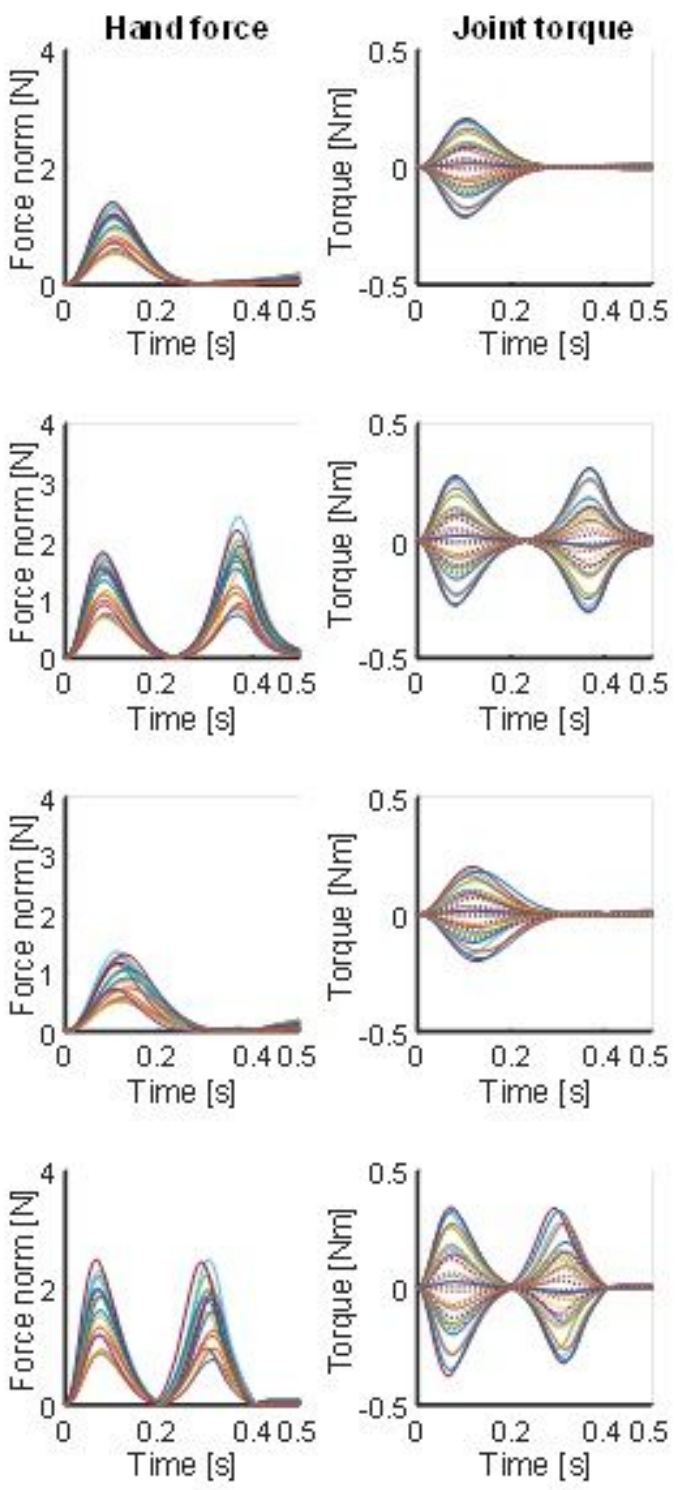

Figure 2 
The centre-out movement for 16 targets. The columns from left to right are the hand paths, hand speed profiles, root mean squared hand force profiles and joint torque profiles. In the hand paths, the target positions are shown as filled circles. In the joint torque profiles, solid and dotted lines indicate the torques of the shoulder and elbow joints, respectively. Cases 1-4 are in rows (a)-(d), respectively.
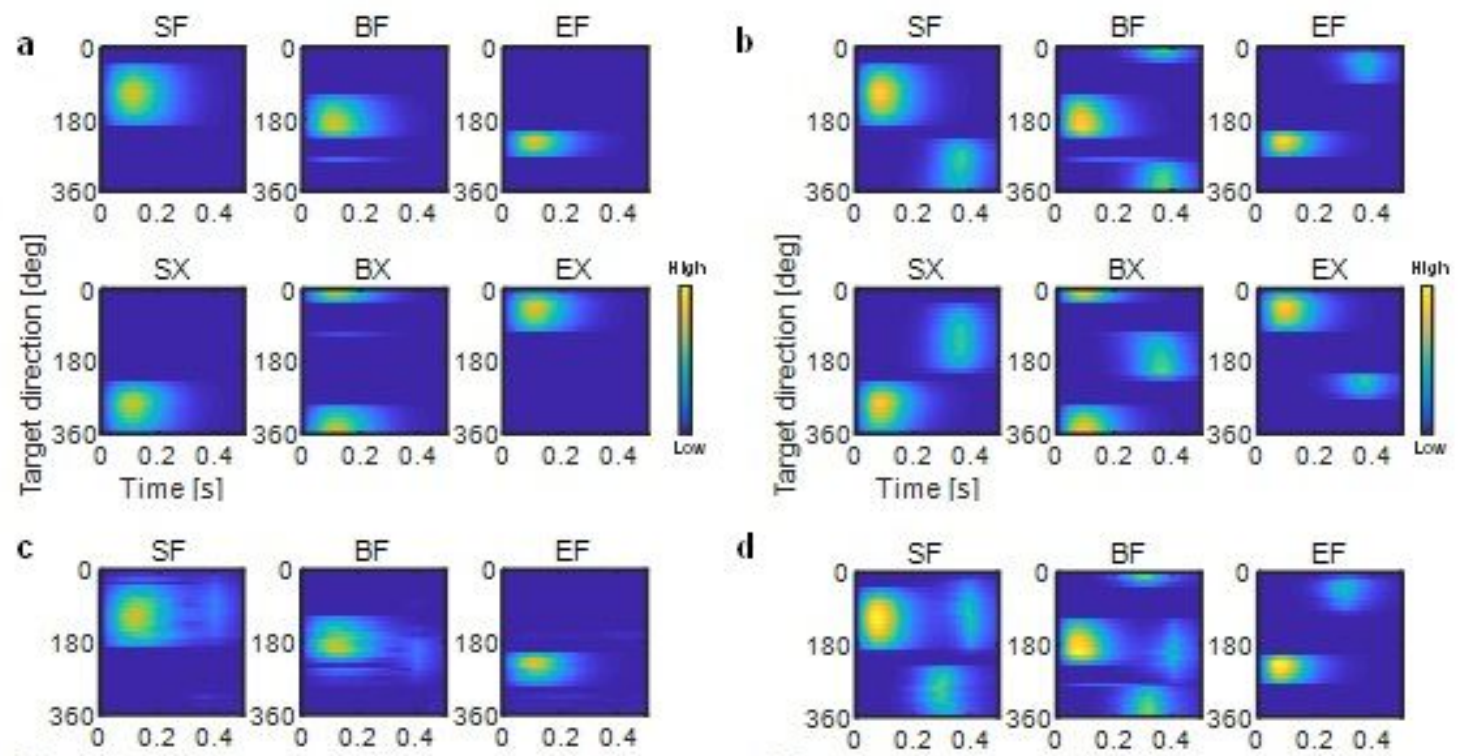

d
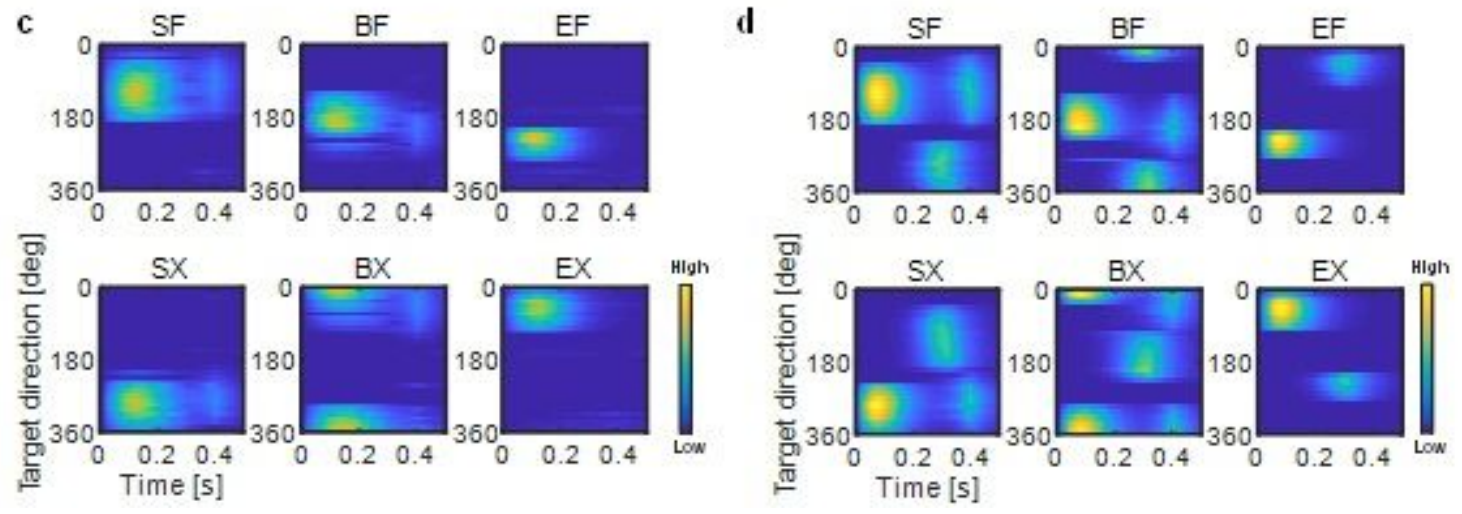

\section{Figure 3}

Muscle activation patterns plotted as a function of time and target direction. Cases 1-4 are shown in (a)-(d), respectively. The values were normalized using the highest activation level in each muscle across all cases. 

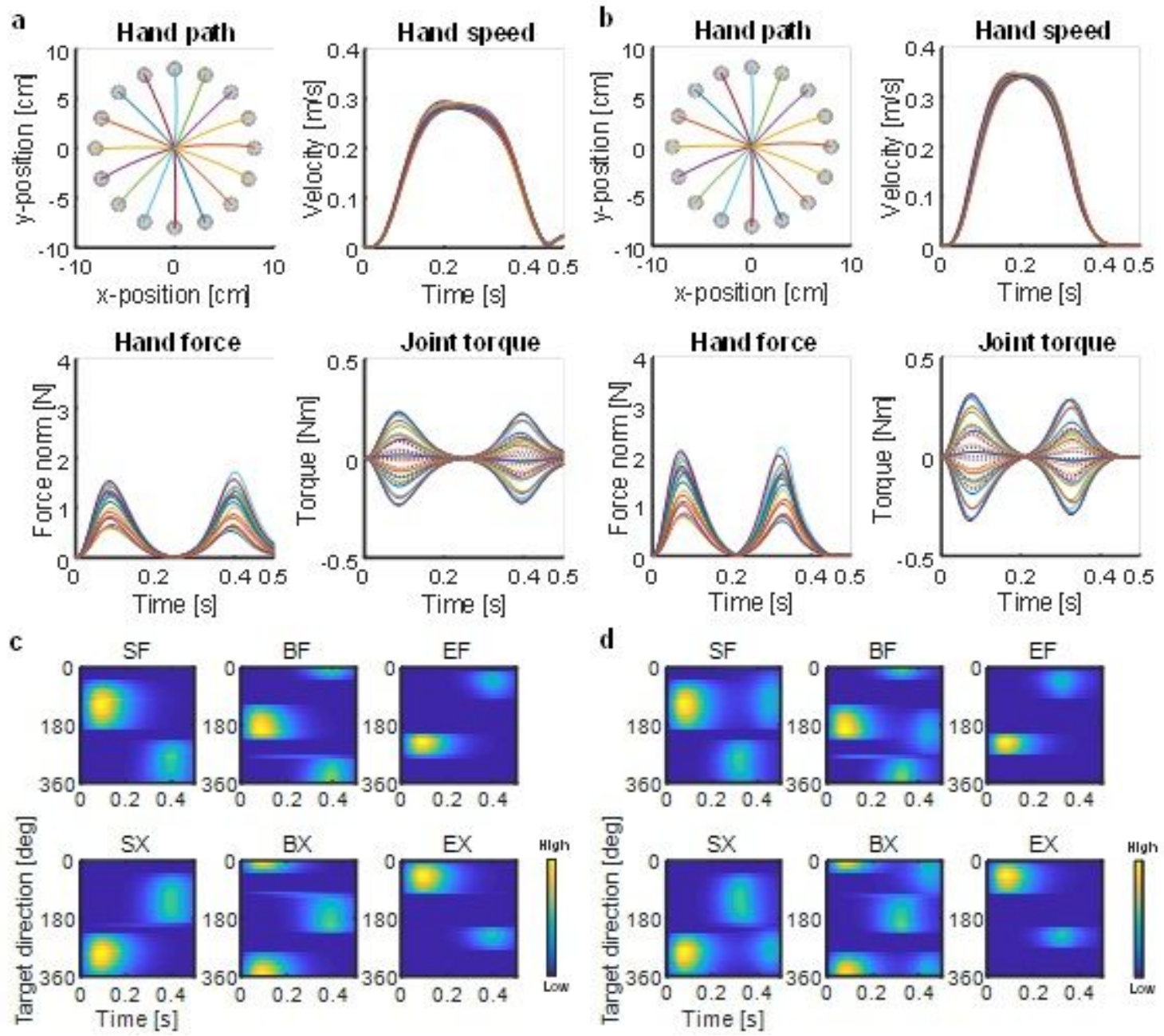

\section{Figure 4}

Stabilization control. The controls of only the position cost, and a pair of the position and velocity costs are shown in $(a, c)$ and $(b, d)$, respectively. $(a, b)$ The centre-out movement for 16 targets. The format is the same as in Figure 2. (c, d) Muscle activation pattern. The format is the same as in Figure 3.
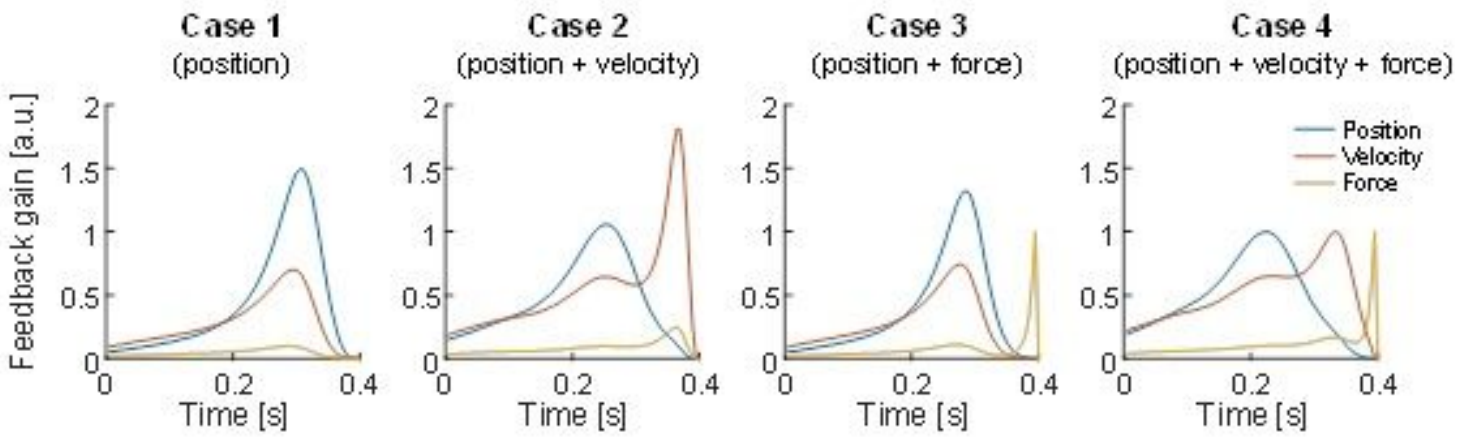

\section{Figure 5}

Typical feedback gains of the optimal feedback control (OFC) model for a linear system. Each of Case 14 corresponds to the same formation of the cost function above results. Each value of the position, velocity and force gains is normalised by that of Case 4 . 

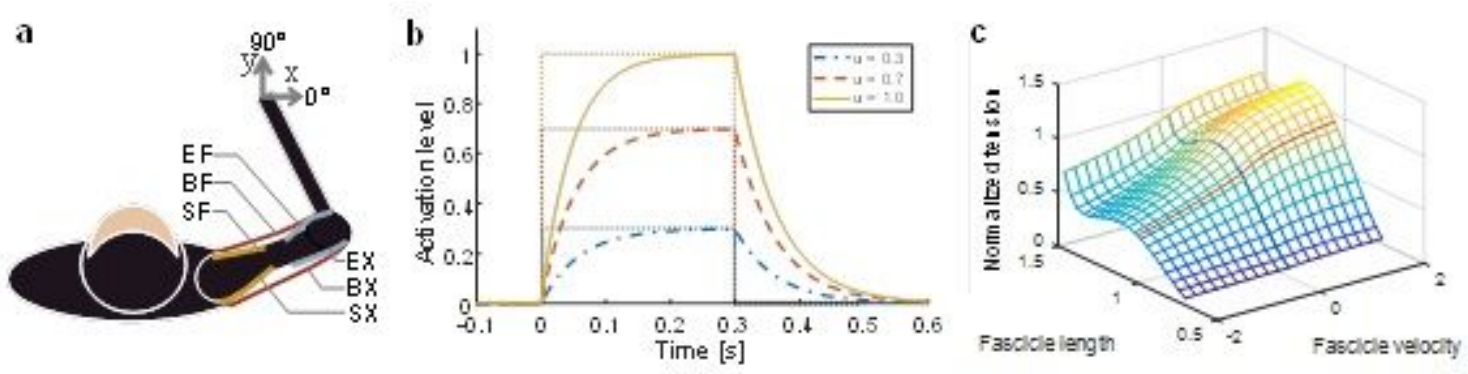

Figure 6

Simulation model. (a) Allocation of muscles in the two-link, six-muscle arm model. The SF models the pectoralis major, coracobrachialis and deltoid anterior muscles. The SX models the posterior and middle deltoid muscles. The BF models the long and short biceps muscles. The BX models the long triceps muscle. The elbow flexor (EF) models the brachialis, brachioradialis and extensor carpi radialis longus muscles. The elbow extensor (EX) models the lateral and medial triceps muscles. (b) Muscle activation dynamics. (c) Length-velocity-tension curve of the muscle model.

\section{Supplementary Files}

This is a list of supplementary files associated with this preprint. Click to download.

- SupplementaryNote.doc

- SupplementaryVideo.mp4 